\title{
MiR-106b-5p Promotes Proliferation and Inhibits Apoptosis by Regulating BTG3 in Non-Small Cell Lung Cancer
}

\author{
Ke Wei Chunfeng Pan ${ }^{a}$ Guoliang Yao ${ }^{b}$ Bin Liuc Teng Mac Yang Xia ${ }^{\mathrm{a}}$ \\ Wei Jiang ${ }^{a} \quad$ Liang Chen $^{\mathrm{a}}$ Yijiang Chen ${ }^{\mathrm{a}}$
}

aDepartment of Thoracic and Cardiovascular Surgery, The First Affiliated Hospital of Nanjing Medical University, Nanjing, 'Department of Cardiothotacic Surgery, The First People's Hospital of Changzhou, The Third Affiliated Hospital of Soochow University, Changzhou, 'Department of Cardiothoracic Surgery, Zhongda Hospital, Southeast University, Nanjing, China

\section{Key Words}

Microrna-106b-5p • BTG3 • Non-small-cell lung cancer $・$ Proliferation • Apoptosis

\begin{abstract}
Background/Aims: MicroRNAs have been validated to play a crucial role in tumorigenesis of non-small cell lung cancer (NSCLC). Although miR-106b-5p has been reported to play a vital role in various malignancies the physiological function of miR-106b-5p in NSCLC still remain unknown. In this study, we investigated the role of miR-106b-5p in NSCLC. Methods: Quantitative real-time polymerase chain reaction was conducted to estimate the expression of miR-106b-5p and BTG3 in both NSCLC tissues and cell lines. The effects of miR-106b$5 p$ on proliferation were determined in vitro using CCK-8 proliferation assays, 5-ethynyl-2'deoxyuridine (EdU) incorporation, colony formation assays and cell-cycle assays and the in vivo effects were evaluated by a mouse tumorigenicity model. Cell apoptosis and cell cycle was investigated by flow cytometric analysis in vitro. The molecular mechanism underlying the relevance between miR-106b-5p and BTG3 was confirmed by luciferase assay and western blot. Results: In current study, we found a relatively higher miR-106b-5p and lower BTG3 expression in NSCLC specimens and cell lines. BTG3 was verified as a direct target of miR$106 b-5 p$ by luciferase assay. In vitro, over-expression of miR-106b-5p promoted proliferation and inhibited apoptosis by down-regulating BTG3 expression. In vivo, miR-106b-5p promoted xenograft tumor formation. Conclusion: Our findings revealed for the first time that miR-106b$5 p$ plays a tumorigenesis role in NSCLC progression by down-regulating BTG3 expression, which may lead to a novel insight to the potential biomarker and novel therapeutic strategies for NSCLC patients.

K. Wei, C. Pan and G. Yao contributed equally to this work.

Yijiang Chen

KARGER 


\section{Introduction}

Lung cancer is one of the most frequent causes of cancer-related morbidity and mortality worldwide [1]. Cancers of the lung and bronchus, colorectum, prostate and breast account for $46 \%$ of all cancer deaths, with more than one-quarter (26\%) due to lung cancer [2]. Lung cancer can be generally divided into two groups: small-cell lung cancer (SCLC, accounting approximately 20\%) and non-small-cell lung cancer (NSCLC, approximately $80 \%$ [ [3]. Thus, NSCLC occupies the vast majority of lung cancer. Although the incidence rate is continuously decreasing after years of studies, the five-year survival rate of NSCLC patients maintains unfavorable due to the lack of early detection methods [2]. Hence, researches on the development and progression of NSCLC would have major implications on the precise diagnosis and novel treatment strategies for patients suffer from lung cancers.

MicroRNAs (miRNAs) are a class of endogenous small non-coding RNAs found in eukaryotes which include 18 to 25 nucleotides and its aberrant expression might cause the degradation of the mRNA or translational inhibition of functional proteins [4-6]. MiRNAs play a significant role in the regulation of cell development, differentiation, proliferation, metastasis, invasion and cell apoptosis [7-14]. Thus, the abnormal alteration of miRNAs could be crucial to the development of diseases including cancers [15-18]. Recent studies have revealed that anomalous expression of miR-106b-5p results in tumorigenesis in diverse cancers including renal cancer, gastric cancer, hepatocellular carcinoma, laryngeal carcinoma, prostate cancer, breast cancer and so on [19-24]. Nonetheless, the biological functions and molecular mechanisms underlying the role of miR-106b-5p in NSCLC are still unidentified.

In the current study, we carried out the luciferase assay and rescue assay and clearly demonstrated that miR-106b-5p acts as an oncogenic miRNA by directly binding to the $3^{\prime}$ UTR of B-cell translocation gene 3 in NSCLC cell lines. BTG3, the protein encoded by this gene is a member of the B-cell translocation gene/transducer of ErbB2 (BTG/Tob (transducer of ERBB2)) anti-proliferative protein family which also includes BTG1, BTG2/PC3/Tis21 (TPAinduced sequence 21), BTG4, Tob1 and Tob2 [25]. Recent researches demonstrate that the family could inhibit cell proliferation and regulate cell-cycle progression and differentiation in a variety of cell types [26, 27]. At present, numerous studies have demonstrated that BTG3 plays a suppressive role in cancer progression and the down-expression of BTG3 could lead to the development of gastric cancer, lung cancer, esophageal adenocarcinoma, and prostate cancer [28-31]. All these results suggest that BTG3 might function as a tumor suppressor in many systems.

In the current study, we expounded the physiology function of miR-106b-5p in NSCLC and demonstrated that BTG3, which is selected by bioinformatics analysis, is a direct target of miR-106b-5p. Through this study we validated for the first time that miR-106b-5p plays a vital role in NSCLC proliferation and apoptosis by regulating BTG3 expression.

\section{Materials and Methods}

Clinical samples

All the NSCLC tissues were derived from patients diagnosed with NSCLC at The First Affiliated Hospital of Nanjing Medical University in 2016. Altogether 75 patients with NSCLC involved in this study had undergone lung tumor resection under the thoracoscopy. Tissue fragments including NSCLC samples and adjacent tissues were immediately frozen in liquid nitrogen during operation and stored at $-80^{\circ} \mathrm{C}$ until RNA extraction. The current study was approved by the Institutional Ethics Committee of the First Affiliated Hospital of Nanjing Medical University and all the specimens were classified according to World Health Organization classification.

Cell culture

H1299, SKMES1, A549, H358, H1299 and16HBE cell lines were obtained from Cell Bank of Type Culture Collection of the Chinese Academy of Sciences (Shanghai, China). Cells were cultured in RPMI-1640 medium 


\section{Cellular Physiology Cell Physiol Biochem 2017;44:1545-1558 \begin{tabular}{l|l|l} 
DOI: 10.1159/000485650 & $\begin{array}{l}\text { O 2017 The Author(s). Published by S. Karger AG, Basel } \\
\text { www.karger.com/cpb }\end{array}$
\end{tabular}}

Wei et al.: MiR-106b-5p in NSCLC

with $10 \%$ fetal bovine serum, $100 \mathrm{U} / \mathrm{ml}$ penicillin and $100 \mu \mathrm{g} / \mathrm{ml}$ streptomycin (Invitrogen, Carlsbad, CA) at $37^{\circ} \mathrm{C}$ in an incubator containing $5 \% \mathrm{CO}_{2}$.

\section{Construction of stable cell lines and Cell transfection}

The target human NSCLC cells H1299 and SPCA1 were transfected with the lentiviral vectors, which were used to construct LV2-hsa-miR-106b-5p-mimic vector (miR-106b-5p) and the LV2-hsa-miR-106b-5pinhibitor vector (anti-miR-106b-5p) (GenePharma, Shanghai, China). The LV2 empty lentiviral construct (NC and anti-NC) served as a negative control. Cells were infected with lentiviruses and screened by puromycin according to standard protocols. For over-expression of BTG3, the stable cell lines were transfected with the BTG3 overexpressing recombinant vector pcDNA3.1-BTG3 (GenePharma, China). For down-regulation of BTG3, small interfering RNAs (siRNA) targeting the encoding region of BTG3 was obtained from GenePharma (Shanghai, China) and the siRNA transfection reagent (Invitrogen) was used according to the manufacturer's instructions. Non-targeting control siRNA was used as negative control.

\section{Isolation of total RNA and quantitative real-time polymerase chain reaction (qRT-PCR)}

Total RNA was isolated from selected cells or clinical specimens using TRIzol reagent (Invitrogen, USA) according to the manufacturer's instructions. MiR-106b-5p real time PCR assays and reverse transcription reactions were performed using miRNA UPL probe assay with Roche UPL probe \#21 (Haoqin, Shanghai, China) in ABI 7900 fast Real-time PCR system (ABI, CA, USA) according to instructions [32].

\section{Colony formation}

A total of $1 \times 10^{3}$ cells were seeded into $60 \mathrm{~mm}$ cell culture dishes. After 10 days, cells were stained with Crystal Violet Staining Solution (Beyotime, Shanghai, China), and colonies containing $\geq 20$ cells were counted. Representative colonies were photographed with a Canon EOS 50.

\section{Cell vitality assay}

Cell Counting Kit-8 (CCK-8) assays were carried out to reveal the effect of miR-106b-5p on cell viability. Transfected Cells $\left(6 \times 10^{3} /\right.$ well $)$ were incubated into 96-well plates and then were stained with 10ul CCK-8 solution (Beyotime, Shanghai, China) per well for 4 hours at $37^{\circ} \mathrm{C}$. The absorbance of each well was measured at $450 \mathrm{~nm}$ (A450) using a spectrophotometer (Thermo Scientific, Rockford, IL, USA).

\section{5-Ethynyl-2'-deoxyuridine (EdU) Proliferation Assay}

To display the function of miR-106b-5p on proliferative, the EdU proliferation assay (RiboBio, Nanjing, China) was carried out according to the manufacturer's instructions. Twenty-four hours after transfection, cells were incubated with $50 \mu \mathrm{M}$ EdU for $2 \mathrm{~h}$. Then an Apollo staining and DAPI staining were performed according to the instructions to detect the EdU positive cells with a fluorescence microscope. The EdU incorporation rate was revealed as the ratio of EdU-positive to total DAPI-positive cells (blue cells).

\section{Flow cytometric analysis}

Apoptosis of the cells was evaluated with Annexin V-FITC/PI Apoptosis Detection Kit (Vazyme, Nanjing, China), keeping with the manufacturer's protocol. The cells were analyzed by using a BD FACSCanto II (BD Biosciences, USA) flow cytometry and the results were analyzed by FlowJo software. In the cell cycle assay, cells were suspended in $70 \%$ cold ethanol overnight after harvest. Then, cells stained with propidium iodide (PI) (Vazyme, Nanjing, China) for 30 minutes were analyzed. The proportion of cells in different cycle phases were calculated and compared.

\section{Bioinformatics analysis}

The Potential targets of miR-106b-5p were predicted and analyzed using bioinformatics method. Four public available algorithms: PicTar, TargetScan, MiRWalk and MiRanda were dovetailed into this study and the results indicated that 3'-UTR of BTG3 binds to miR-106b-5p with the high score, suggesting BTG3 might be a potential target of miR-106b-5p.

\section{Luciferase report assay}

Human BTG3 3'-UTR sequence or the mutant sequence of BTG3 3'-UTR with the predicted target sites was inserted into pGL3 promoter vector (Genscript, Nanjing, China). Both H1299 and SPCA1 cells were planted onto 24-well plates $\left(5 \times 10^{5}\right.$ cells/well) the day before transfection and were co-transfected with luciferase reporter vectors $(0.12 \mu \mathrm{g})$ and $40 \mathrm{nM}$ of miR-106b-5p mimic or negative control oligoribonucleotides by using Lipofectamine 2000 (Invitrogen). Luciferase report assay was measured in Victor 1420 Multilabel 
Counter (Wallac, Finland) by using Luciferase Assay System (Promega, USA) according to the standard protocol.

\section{Western blotting}

Cells were lysed in ice-cold RIPA buffer (Beyotime, Shanghai, China) with 10nM PMSF for 30 minutes and then collected to extract total protein. Total Proteins lysates were fractionated by sodium dodecyl sulfate polyacrylamide gel electrophoresis (SDS-PAGE) and then were transferred to polyvinylidene fluoride (PVDF) membrane. Rabbit anti-GAPDH monoclonal antibody was taken as control to estimate protein loading. The membrane was blocked in 5\% non-fat milk in mixture of tris-buffered saline (TBS) and Tween 20 (TBST) for $2 \mathrm{~h}$ at room temperature and then immunostained overnight at $4{ }^{\circ} \mathrm{C}$ using rabbit anti-BTG3 (1:1000, Cell Signaling Technology, CST, USA). Relative protein levels were quantified by ImageJ software.

\section{In vivo tumor xenograft model}

All animal experiments were approved by the Animal Care and Use Committee of Nanjing Medical University. For the in vivo tumor growth studies, twenty 2-month-old female BALB/c nude mice were purchased from the Animal Center of NJMU and were divided into 4 groups ( $\mathrm{n}=5$ per group). Then were injected subcutaneously in the left flank with $1 \times 10^{6}$ tumor cells stably transfected with Lv-NC or Lv-mir106b-5p or Lv-anti-miR-106b-5p or Lv-anti-NC. All mice were monitored every 7 days and were sacrificed 3 weeks later. Tumor volume was evaluated with the following formula: tumor volume $=$ volume $=\left(\right.$ width $^{2}$ $\times$ length) $/ 2$.

\section{Immunohistochemistry analysis of subcutaneous xenograft model}

All implanted tumors were fixed in $4 \%$ formalin and then embedded in paraffin. After blocking endogenous peroxides and proteins, sections (thickness, $4 \mu \mathrm{m}$ ) were incubated overnight at $4{ }^{\circ} \mathrm{C}$ with primary antibodies for specific detection of BTG3 or Ki-67 (Maixin Bio, China). After washing with PBS, sections were incubated with HRP-Polymer-conjugated secondary antibody at $37^{\circ} \mathrm{C}$ for $1 \mathrm{~h}$. Subsequently, sections were stained with 3, 3-diaminobenzidine solution for $3 \mathrm{~min}$ and the nuclei were counterstained with hematoxylin. Tumor sections were examined in a blinded manner. The percentage of positive tumors

Table 1. Expression of miRNA-106b-5p expression and BTG3 in human NSCLC according to patients' clinicopathological characteristics

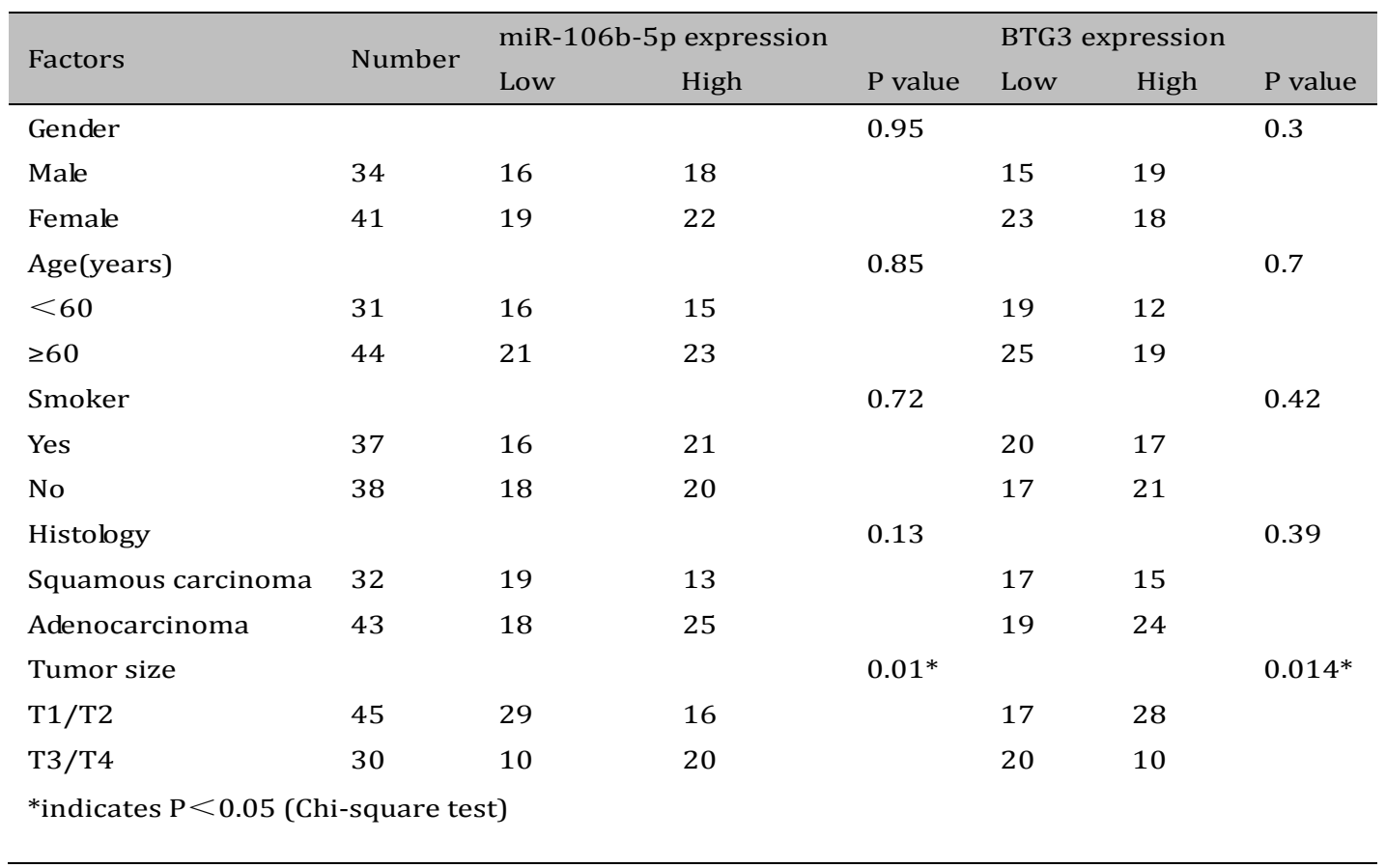




\section{Cellular Physiology Cell Physiol Biochem 2017;44:1545-1558

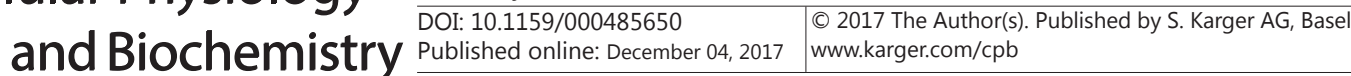 \\ Wei et al.: MiR-106b-5p in NSCLC}

and cell-staining intensity was determined based on three randomly selected fields for each section. Samples were observed with an Olympus microscope (Olympus, Tokyo, Japan).

\section{Statistical analysis}

The chi-squared test was used to test the significance of differences in the data of Table. 1. Pearson correlation was used for correlation analysis in Fig. 1C. All experiments were performed in triplicate independently. The data were expressed as mean \pm standard deviation (SD). Student's unpaired t-test was used to determine the significant differences of other results. $\mathrm{P}<0.05$ was considered to be statistically significant. In this study, '*' indicates ' $\mathrm{P}<0.05$ ', '**'indicates ' $\mathrm{P}<0.01$ ', and '***' indicates ' $\mathrm{P}<0.001$ '. All analysis was performed using SPSS 19.0 software.

\section{Results}

High expression level of miR-106b-5p were inversely correlated with BTG3 expression in human NSCLC tissues and cell lines

To determine the expression level of miR-106b-5p and BTG3 in NSCLC tissues and paired adjacent normal tissues, qRT-PCR was carried out. As shown in Fig. 1A and B, comparing to corresponding adjacent normal tissues, miR-106b-5p was markedly up-regulated in NSCLC samples while BTG3 had a significantly lower expression. To evaluate the correlation between miR-106b-5p and BTG3, we implemented a two-tailed Pearson's correlation analysis. It turned out that the expression of miR-106b-5p was negatively correlated with BTG3 expression (Fig. 1C). Through the data analysis of the clinical features, we discovered that miR-106b-5p and BTG3 had a correlation with the development of NSCLC (Table 1). Further in this study, we determined the expression level of miR-106b-5p and BTG3 in five lung cancer cell lines (H1299, SKMES1, A549, H358 and SPCA1) and one normal human bronchoepithelial cell line (16HBE) via qRT-PCR. The result showed that miR-106b-5p had a high-level expression while BTG3 expression decreased when compared with 16HBE cell line (Fig. 1D, E). Combination of the above consequences validated that miR-106b-5p expression was inversely correlated to the expression of BTG3 and both of them may make a difference in the progression of NSCLC.

\section{MiR-106b-5p promotes cell proliferation of NSCLC in vitro}

According to the miR-106b-5p expression level in NSCLC cell lines, we selected H1299 and SPCA1 cells for transfection with lentiviral constructs which were designed to overexpress or knockdown miR-106b-5p (SPCA1-miR-106b-5p, SPCA1-NC, H1299-anti-miR-106b-5p and H1299-anti-NC). The transfection efficiency of the cell lines was validated via qRT-PCR (Fig. $2 A)$. In order to determine the effect of miR-106b-5p on proliferation in NSCLC, we carried out CCK-8 assay, EdU incorporation assay and used the colony formation assay for evaluating the influence on proliferation. The results of CCK-8 assay turned out that overexpression miR-106b-5p accelerated proliferation when compared with the control group. In contrast, down-regulated miR-106b-5p showed inverse performance (Fig. 2B). After that, as shown in Fig. 2C, results indicated that miR-106b-5p promotes colony formation ability while opposite influence emerged in knockdown group. Besides, EdU incorporation assay was employed for further assessment of the impact of miR-106b-5p on proliferation. The EdU incorporation assay revealed distinct difference on proliferation in lentivirus transfected H1299 and SPCA1 cell lines (Fig. 2D). Combining with above analyses, miR-106b-5p might play a pivotal role in promoting proliferation in NSCLC cells in vitro.

MiR-106b-5p inhibits apoptosis and promotes cell cycle in NSCLC cells

To investigate the role of miR-106b-5p in NSCLC progression, Flow cytometric analysis was adopted to examine if miR-106b-5p affects apoptosis or cell cycle. Cell apoptosis is an important segment in NSCLC tumorigenesis. Hence, flow cytometric analysis of apoptosis was performed to elucidate whether miR-106b-5p functions as a candidate carcinogenic gene in NSCLC. Overexpression of miR-106b-5p remarkably suppressed apoptosis in SPCA1 
Fig. 1. Expression of miR-106b-5p and BTG3 in NSCLC clinical samples $(\mathrm{N}=75)$ and cell lines. A: The expression levels of miR-106b-5p in 75 pairs of human NSCLC tissues and non-NSCLC tissues were explored using miRNA RT-PCR. B: The mRNA expression level of BTG3 relative to GAPDH in human NSCLC tissues and corresponding adjacent tissues were detected by using qRT-PCR. C: A negative correlation was found between RNA expression of miR106b-5p and BTG3 in

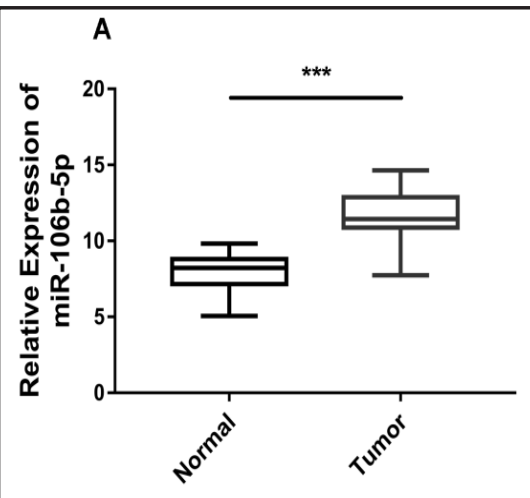
B
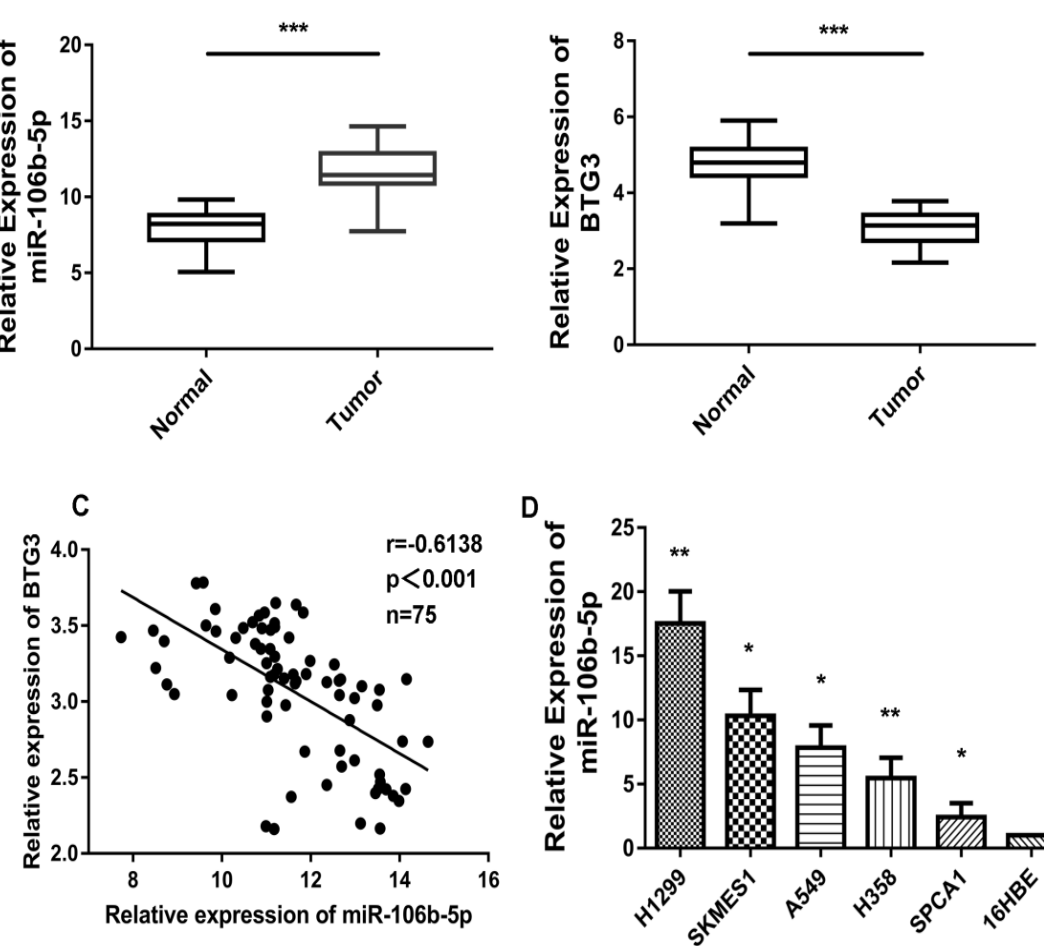

tumor samples. D and E: Expression levels of miR-106b-5p and BTG3 in normal lung cell (16HBE) and 5 lung cancer cell lines (H1299, SKMES1, A549, H358, SPCA1). Data are represented as mean \pm SD. *indicates $\quad \mathrm{p}<0.05$, ${ }^{* *} \mathrm{p}<0.01,{ }^{* * *} \mathrm{p}<0.001$.
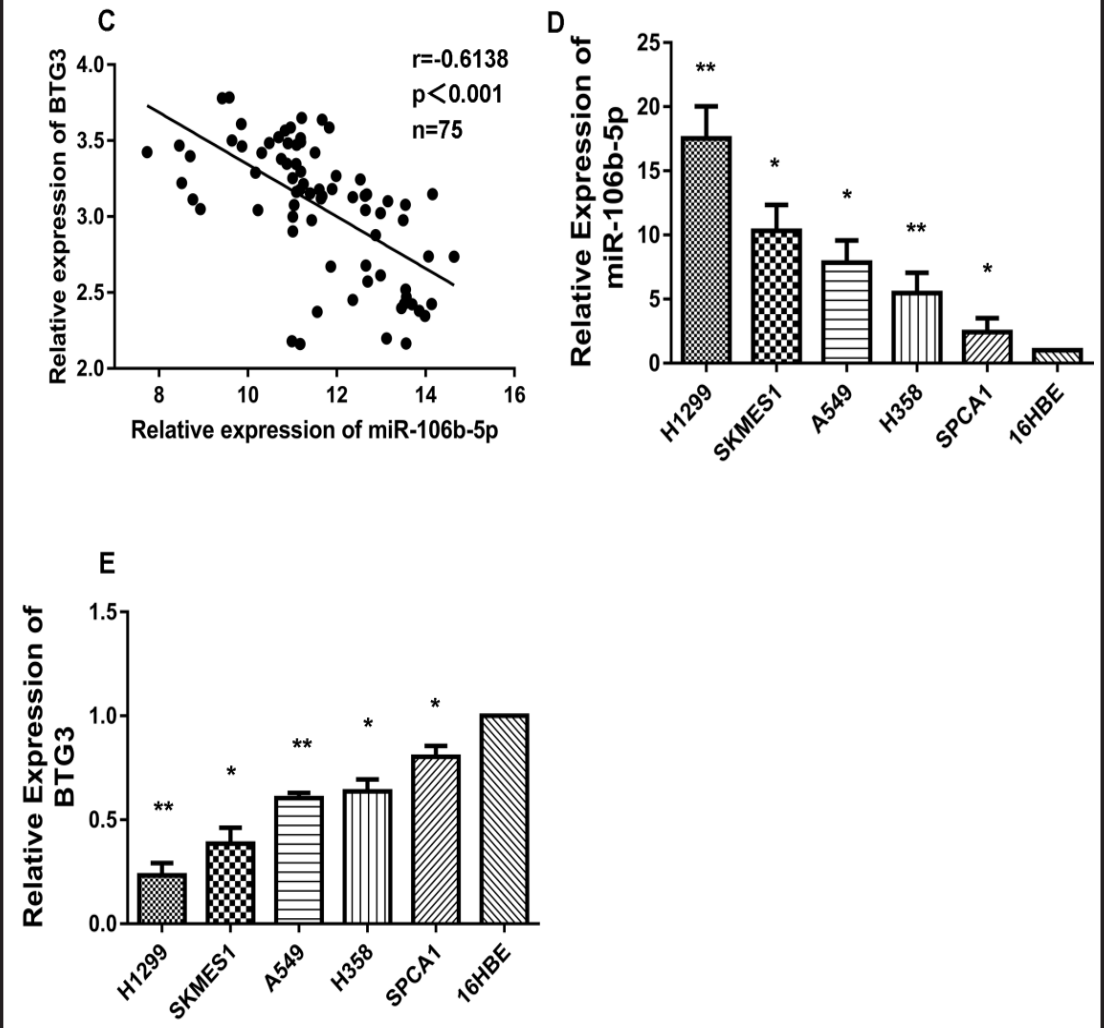

cells while down-regulation of miR-106b-5p induced an increase in apoptotic death in H1299 cells (Fig. 2E). Furthermore, in order to delineate the correlation between cell cycle variability and proliferation in NSCLC cells, cell cycle of lentivirus transfected cells was measured by flow cytometry. As shown in Fig. 2F, up-regulated miR-106b-5p promoted cell cycle and resulted in a decrease in the G0/G1 fraction in SPCA1 cells. Conversely, downregulated miR-106b-5p exhibited an increase trend in the cell population in the G0/G1 phase. These findings suggested that miR-106b-5p inhibits cell apoptosis and accelerates cell cycle in NSCLC cells.

MiR-106b-5p suppress BTG3 expression via interacting directly with a hypothetic binding site of BTG3-3'-UTR

To explore the potential target of miR-106b-5p, publicly available database (MiRanda, MiRWalk, PicTar and TargetScan) were adopted. A putative binding site of BTG3-3'-UTR for miR-106b-5p was identified (mirSVR score: -1.3608). To verify the accuracy of the prediction, we applied dual luciferase reporter assays. On the basis of prediction, the wild type miR106b-5p target sequences of BTG3-3'-UTR or mutant miR-106b-5p target sequences of 


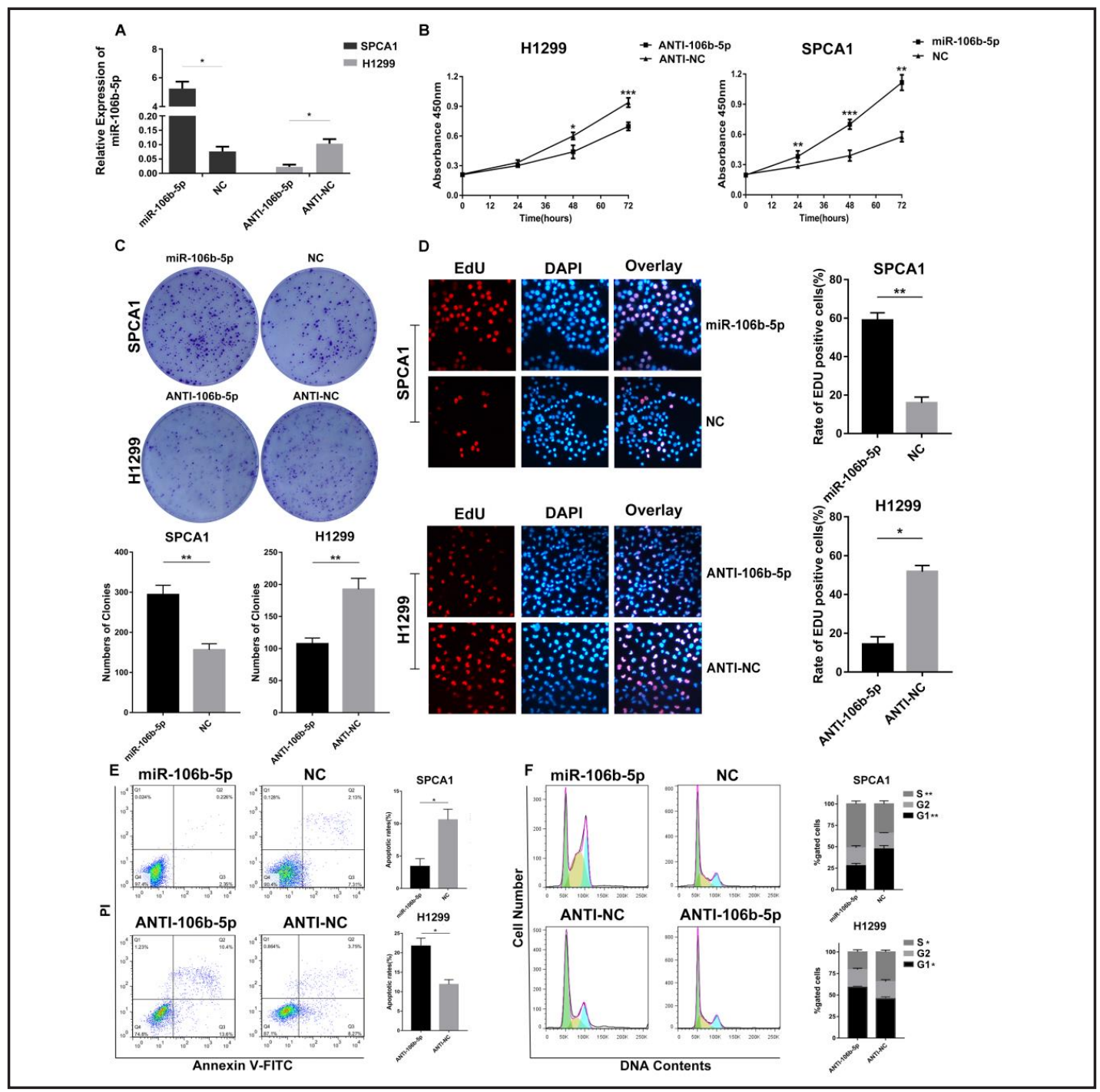

Fig. 2. MiR-106b-5p promotes cell proliferation and inhibits cell apoptosis. A: The results of miR-106b$5 p$ expression in cell lines transfected with miR-106b-5p-mimics and miR-106b-5p-inhibitor lentivirus respectively were validated by using qRT-PCR. B: By cck-8 assay, we found distinct differences on proliferation after manipulation of miR-106b-5pmimics and miR-106b-5p-inhibitor lentivirus at 24-h, 48-h and 72-h time points. NC and anti-NC were used as controls. C: Effects of miR-106b-5p alteration on the colony formation of NSCLC cells. D: Representative profiles of Edu cell growth in SPCA1 cells and H1299 cells after transfection with miR-106b-5p-mimics and miR-106b-5p-inhibitor respectively compared with the control. E: Effects of miR-106b-5p alteration on cell cycle distribution of NSCLC cells. F: FACS analysis of the effect of miR-106b-5p expression alteration on cell apoptosis. ${ }^{*} \mathrm{p}<0.05,{ }^{* *} \mathrm{p}<0.01,{ }^{* * *} \mathrm{p}<0.001$. All experiments are performed three times independently. The data expressed as the mean \pm SD.

BTG3-3'-UTR was cloned into pGL3 luciferase reporter vector (PGL3-BTG3, PGL3-BTG3MUT). H1299 and SPCA1 cells were co-transfected with PGL3-BTG3 or PGL3-BTG3-MUT and miR-106b-5p mimics or its negative control. Results from luciferase reporter assay showed that Luciferase activity of wild-type BTG3 reporter vector was significantly decreased after co-transfection with the miR-106b-5p mimics. Nevertheless, luciferase activity of mutant reporter vector was not affected (Fig. 3A). In addition, to examine the expression of BTG3 in established stable transfection H1299 and SPCA1 cells we performed qRT-PCR and western blot assay. It turned out that miR-106b-5p overexpression down-regulated the expression of

\section{KARGER}




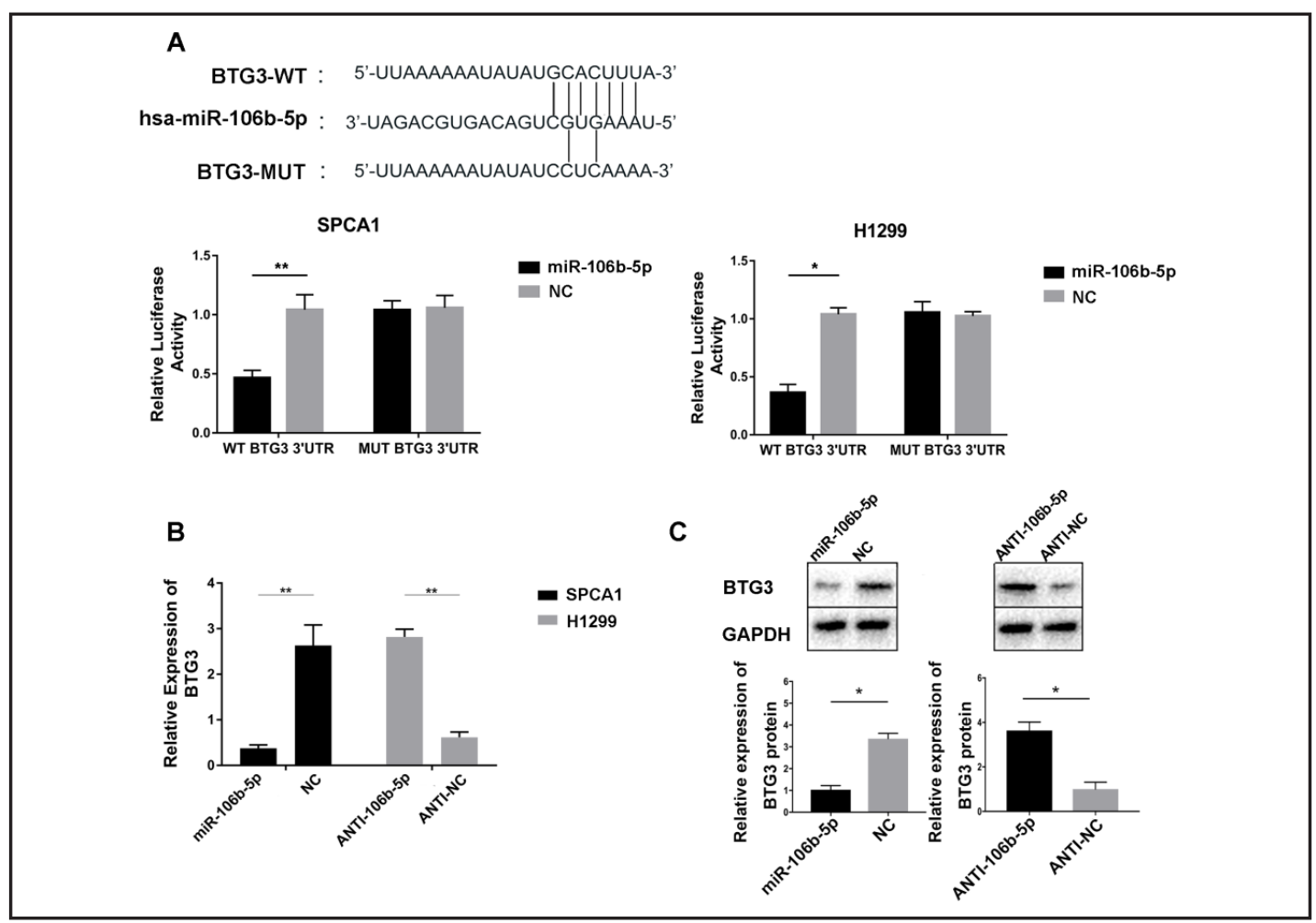

Fig. 3. MiR-106b-5p regulates BTG3 expression by directly binding its 3'-UTR. A: Luciferase reporter assay was conducted to verify that miR-106b-5p directly bound to the 3'-UTR region of BTG3. Luciferase activity was analyzed in cells co-transfected with miR-106b-5p-mimics or negative control with pGL3-BTG3-WT or pGL3-BTG3-MUT. B: BTG3 mRNA expression levels in transfected SPCA1 and H1299 cells were analyzed by qRT-PCR. GAPDH was used as a control. C: BTG3 protein expression levels in transfected SPCA1 and H1299 cells were analyzed by western blot. GAPDH was used as a control. Data are represented as mean \pm SD. ${ }^{*}$ Indicates $\mathrm{P}<0.05,{ }^{* *} \mathrm{p}<0.01,{ }^{* * *} \mathrm{p}<0.001$.

BTG3 (Fig. 3B, C). The findings suggested BTG3 served as a functional target of miR-106b-5p and BTG3 was negatively regulated by miR-106b-5p.

MiR-106b-5p promotes proliferation and inhibits apoptosis in NSCLC cells by regulating BTG3

To illuminate whether the alteration of proliferation and apoptosis induced by aberrant expression of miR-106b-5p was in connection with the expression of BTG3 in NSCLC cells, we conducted rescue assays. For down-regulating BTG3 expression we used the RNA interference (small interfering RNA (siRNA)) technology in H1299 cells. SPCA1 cells were transfected with pcDNA3.1-BTG3 for up-regulating BTG3 expression. The expression of BTG3 on both mRNA and protein levels in transfected cells were examined by qRT-PCR and western blot analysis respectively. Compared with control group, miR-106b-5p down-expression H1299 cells cotransfected with si-BTG3 exhibited a distinctly lower expression level of BTG3. Consistently, relative expression level of BTG3 increased in miR-106b-5p up-expression SPCA1 cells cotransfected with pcDNA3.1-BTG3 (Fig. 4A, B). Subsequently, the colony formation assay, CCK8 assay and EdU incorporation assay were adopted in co-transfected cells. As shown in Fig. 4C, D, E, down-regulation of BTG3 effectively reversed the suppressing effect of miR-106b-5p down-expression on proliferation in $\mathrm{H} 1299$ cells. Similarly, the promoting effect of miR-106b$5 p$ over-expression on proliferation was counteracted by BTG3 up-regulation in SPCA1 cells (Fig. 4C, D and E). Furthermore, we performed Flow cytometric analysis to explore whether the effects of miR-106b-5p on cell cycle and apoptosis were mediated by regulation of BTG3 in NSCLC cells. It turned out that down-expression of BTG3 inhibited apoptosis and removed 


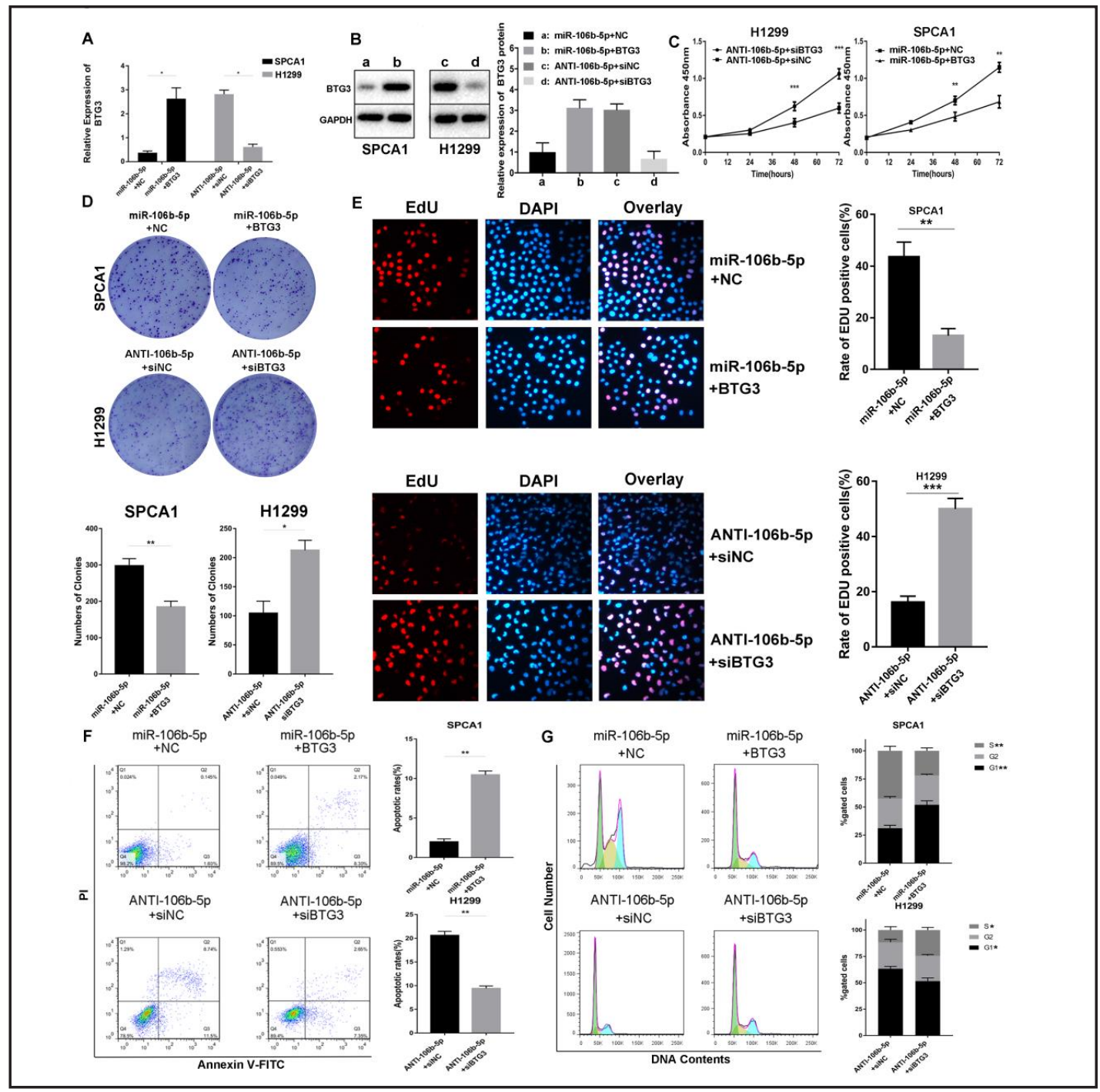

Fig. 4. MiR-106b-5p promotes proliferation and inhibits apoptosis in NSCLC cells by regulating BTG3. A: The expression of BTG3 was verified by qRT-PCR in co-transfected cell lines. B: Western blot was used to verify the expression of BTG3. C: CCK-8 was used to determine the proliferation of NSCLC cells co-transfected with miR-106b-5p mimics and miR-106b-5p-inhibitor lentivirus. D, E: The roles of miR-106b-5p and BTG3 in the regulation of NSCLC cell proliferation and apoptosis were examined by colony formation assay and the EdU assay. The rescue experiments for miR-106b-5p overexpression were performed by ectopic expression of BTG3 without its 3'-UTR in SPCA1 cells. Similar rescue experiments for miR-106b-5p silencing were performed by down-regulation of BTG3 in H1299 cells. F, G: The roles of miR-106b-5p and BTG3 in the regulation of NSCLC cell apoptosis and cycle were verified by flow cytometry. The rescue experiments for miR-106b-5p overexpression were performed by ectopic expression of BTG3 without its 3'-UTR in SPCA1 cells. Similar rescue experiments for miR-106b-5p silencing were performed by down-regulation of BTG3 in $\mathrm{H} 1299$ cells. The data came from at least three independent experiments. A representative data set is displayed as mean \pm SD values. ${ }^{*} \mathrm{p}<0.05,{ }^{* *} \mathrm{p}<0.01,{ }^{* * *} \mathrm{p}<0.001$.

the effect of cell cycle arrest in miR-106b-5p down-expression H1299 cells. Likewise, upexpression of BTG3 motivated apoptosis and induced cell-cycle arrest in the G0/G1 phase in miR-106b-5p up-expression SPC-A1 cells (Fig. 4F, G). These findings suggested that miR106b-5p promotes proliferation and inhibits apoptosis in NSCLC cells by targeting BTG3. 


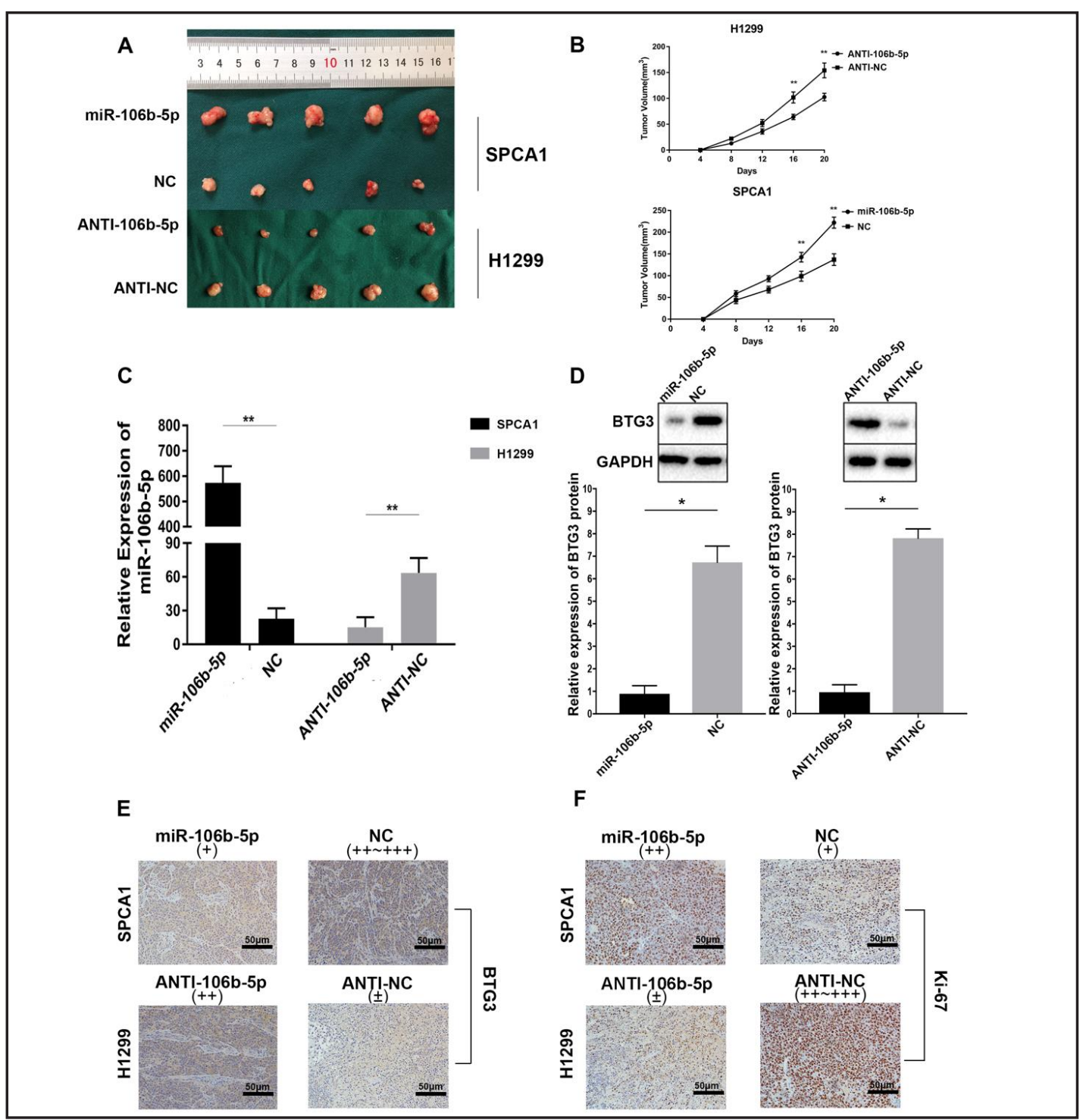

Fig. 5. MiR-106b-5p promotes tumorigenicity in vivo. A: Photographs of tumors obtained from the different groups of nude mice transfected with miR-106b-5p-mimics and miR-106b-5p-inhibitor, respectively. B: Growth curve of tumor volumes were calculated. Data are shown as mean \pm SD. Tumor volumes between miR-106b-5p-mimics or miR-106b-5p-inhibitor and corresponding control group was significantly different at $\mathrm{p}<0.01$ by two-way ANOVA. C-E: The expression levels of miR-106b-5p and BTG3 protein in the implanted tumors that were transfected with miR-106b-5p-mimics and miR106b-5p-inhibitor were explored by miRNA RT-PCR, Western blot and immunohistochemistry staining. F: Immunohistochemical staining against Ki-67 assay were used to determine the effects of miR-106b-5p expression alteration on cell proliferation in the samples collected from nude mice. Scale bar is $50 \mu \mathrm{m}$. ${ }^{*} \mathrm{p}<0.05,{ }^{* *} \mathrm{p}<0.01,{ }^{* * *} \mathrm{p}<0.001$. The data expressed as the mean \pm SD.

\section{MiR-106b-5p promotes xenograft tumor formation}

To further investigate the role of miR-106b-5p in the proliferation of NSCLC cells in vivo, H1299 cells stably down-regulating miR-106b-5p and SCPA1 cells stably up-regulating miR-106b-5p were orthotopically inoculated into each nude mice as well as scramble in the left flank of the nude mice. As shown in Fig. 5A, B, mice inoculated with miR-106b-5p overexpression SPCA1 cells had increased tumor volume compared with those in the negative group. As expected, the tumor volume of anti-miR-106b-5p group was decreased compared

\section{KARGER}


with those in the anti-NC group. Furthermore, miRNA RT-PCR revealed that miR-106b-5p expression significantly increased in miR-106b-5p overexpressing group compared with the control group and the opposite expression level of miR-106b-5p was observed in antimiR-106b-5p group (Fig. 5C). Reversely, BTG3 expression decreased in miR-106b-5p upregulating group and increased in miR-106b-5p down-regulating group. Then BTG3 protein expression level was examined by western blotting and immunohistochemical analyses. As shown in the Fig. 5D-E, BTG3 protein expression was dramatically reduced in miR-106b$5 p$ over-expression group and significantly increased in miR-106b-5p down-expression group compared with the control group respectively. The results of Immunohistochemical staining against Ki-67 assay are consistent with the findings that miR-106b-5p promotes the proliferation of NSCLC (Fig. 5F). These observations revealed that miR-106b-5p promotes tumor formation in vivo.

\section{Discussion}

Lung cancer remain the one of the most common cancer worldwide and among the developing countries, especially China, lung cancer turn into the most common cancer and the leading cause of death by cancer [33]. Researches on novel biomarkers and new therapeutic targets are particularly critical. Herein, we provide the evidence that miR106b-5p promotes proliferation and inhibits apoptosis in NSCLC cells by regulating BTG3 expression. All the experiment data indicate that miR-106b-5p might play a pivotal role in the progression of NSCLC.

Aberrant expression of miRNAs is correlated with cancer development, including migration and proliferation [34-38]. MiR-106b-5p is located to human chromosome 7q21 and transcribed from the miR-106b-25 cluster $[17,19]$. To date, a number of experiments have been designed to explore the role of miR-106b-5p in the development of various cancers [15, 16]. Over-expression of miR-106b-5p in hepatocellular carcinoma promoted cell migration and metastasis by activating epithelial-mesenchymal transition [39]. MicroRNA-106b-5p played an oncogenic role in glioma by targeting RBL1, RBL2 and CASP8 [40]. In breast cancer, miR-106b-5p was reported to promote cell migration, invasion, and proliferation by targeting FUT6[41]. It's over-expression in colorectal cancer promoted cell migration and invasion by directly targeting DLC1 [42]. And MicroRNA-106b-5p was also certified as a suppressor in expression of SETD2 and plays an important role in regulating ccRCC cell proliferation and apoptosis through SETD2-dependent way [43]. Nevertheless, to our knowledge, the function of miR-106b-5p in NSCLC cells still remains unclear. Therefore, we revealed for the first time that miR-106b-5p promotes proliferation and inhibits apoptosis in NSCLC cells.

BTG3 was separated by low-stringency screening of a cDNA library using BTG1 and BTG2/TIS21 probes [44]. Some evidence has shown that BTG3 expression is associated with tumorigenesis and serves as a candidate tumor suppressor gene in various cancers [4547]. As reported, BTG3 involves in the suppression of proliferation, cell cycle progression and metastasis [48-51]. However, the association between BTG3 and miRNA has rarely been reported. Here, based on the data of luciferase assay, we revealed that BTG3 is a direct downstream target of miR-106b-5p. According to the rescue assay, the physiological function of over-expression miR-106b-5p was totally abrogated by re-expression of BTG3. Thus, we consider that miR-106b-5p function as a carcinogenic role by regulating the expression of BTG3 in the progression of NSCLC.

\section{Conclusion}

In summary, our research validated for the first time that miR-106b-5p played a crucial role in NSCLC proliferation and apoptosis by regulating BTG3 expression. However, owing to the limitation of the number of NSCLC samples and cell types, the remaining biological function of miR-106b-5p in NSCLC tumorigenesis and the further mechanism underlying the relevance between miR-106b-5p and BTG3 need more elaborate studies to farther expound. 
These findings may provide a better understanding of the function of miR-106b-5p in NSCLC progression and put forward a potential biomarker and novel therapeutic strategy for NSCLC patients.

\section{Abbreviations}

NSCLC (non-small cell lung cancer), miRNA (microRNA), BTG3 (B-cell translocation gene 3), PCR (Polymerase chain reaction), 3'-UTR (3'-untranslated region), siRNA (small interfering RNA), CCK-8 (cell-counting kit-8 assay), DAPI (4',6-diamidino-2-phenylindole), FBS (fetal bovine serum), PBS (phosphate buffered solution).

\section{Acknowledgements}

This work was supported by The National Natural Science Foundation of China (grant no. 81572263) and the Science and Technology Project of Jiangsu Provincial Health Department (grant no. H200821).

\section{Disclosure Statement}

None.

\section{References}

$>1$ Torre LA, Bray F, Siegel RL, Ferlay J, Lortet-Tieulent J, Jemal A: Global cancer statistics, 2012. CA Cancer J Clin 2015;65:87-108.

-2 Siegel RL, Miller KD, Jemal A: Cancer Statistics, 2017 CA Cancer J Clin 2017;67:7-30.

-3 Gompelmann D, Eberhardt R, Herth FJ: Advanced malignant lung disease: what the specialist can offer. Respiration 2011;82:111-123.

4 Johnson AM, Hines RB, Johnson JA, 3rd, Bayakly AR: Treatment and survival disparities in lung cancer: the effect of social environment and place of residence. Lung Cancer 2014;83:401-407.

5 Bartel DP: MicroRNAs: genomics, biogenesis, mechanism, and function. Cell 2004;116:281-297.

6 Zhu X, Fu C, Zhang L, Xu G, Wang S: MiRNAs associated polymorphisms in the 3'UTR of MET promote the risk of non-small cell lung cancer. Cell Physiol Biochem 2015;37:1159-1167.

7 Ambros V: The functions of animal microRNAs. Nature 2004;431:350-355.

8 Li J, Yu T, Cao J, Liu L, Liu Y, Kong HW, Zhu MX, Lin HC, Chu DD, Yao M, Yan MX: MicroRNA-148a Suppresses Invasion and Metastasis of Human Non-Small-Cell Lung Cancer. Cell Physiol Biochem 2015;37:1847-1856.

-9 Kurashige J, Kamohara H, Watanabe M, Hiyoshi Y, Iwatsuki M, Tanaka Y, Kinoshita K, Saito S, Baba Y, Baba H: MicroRNA-200b regulates cell proliferation, invasion, and migration by directly targeting ZEB2 in gastric carcinoma. Ann Surg Oncol 2012;19:S656-664.

10 Gao F, Zhao ZL, Zhao WT, Fan QR, Wang SC, Li J, Zhang YQ, Shi JW, Lin XL, Yang S, Xie RY, Liu W, Zhang TT, Sun YL, Xu K, Yao KT, Xiao D: miR-9 modulates the expression of interferon-regulated genes and MHC class I molecules in human nasopharyngeal carcinoma cells. Biochem Biophys Res Commun 2013;431:610-616.

11 He Z, Xia Y, Pan C, Ma T, Liu B, Wang J, Chen L, Chen Y: Up-Regulation of MiR-1111 Inhibits Metastasis of Non-Small Cell Lung Cancer by Regulating BMI1. Cell Physiol Biochem 2015;37:387-398.

12 Jemal A, Bray F, Center MM, Ferlay J, Ward E, Forman D: Global cancer statistics. CA Cancer J Clin 2011;61:69-90.

13 Deng W, Yan M, Yu T, Ge H, Lin H, Li J, Liu Y, Geng Q, Zhu M, Liu L, He X, Yao M: Quantitative proteomic analysis of the metastasis-inhibitory mechanism of miR-193a-3p in non-small cell lung cancer. Cell Physiol Biochem 2015;35:1677-1688. 


\section{Cellular Physiology Cell Physiol Biochem 2017;44:1545-1558

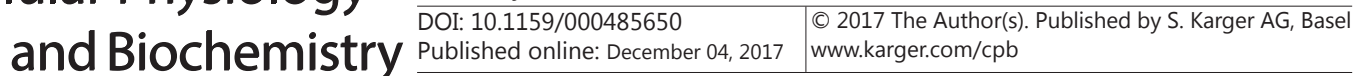

Wei et al.: MiR-106b-5p in NSCLC

14 Niu X, Liu S, Jia L, Chen J: Role of MiR-3619-5p in beta-Catenin-Mediated Non-Small Cell Lung Cancer Growth and Invasion. Cell Physiol Biochem 2015;37:1527-1536.

15 Calin GA, Sevignani C, Dumitru CD, Hyslop T, Noch E, Yendamuri S, Shimizu M, Rattan S, Bullrich F, Negrini M, Croce CM: Human microRNA genes are frequently located at fragile sites and genomic regions involved in cancers. Proc Natl Acad Sci U S A 2004;101:2999-3004.

16 Guo M, Zhang X, Wang G, Sun J, Jiang Z, Khadarian K, Yu S, Zhao Y, Xie C, Zhang K, Zhu M, Shen H, Lin Z, Jiang C, Shen J, Zheng Y: miR-603 promotes glioma cell growth via Wnt/beta-catenin pathway by inhibiting WIF1 and CTNNBIP1. Cancer Lett 2015;360:76-86.

-17 Li T, Xie J, Shen C, Cheng D, Shi Y, Wu Z, Deng X, Chen H, Shen B, Peng C, Li H, Zhan Q, Zhu Z: Upregulation of long noncoding RNA ZEB1-AS1 promotes tumor metastasis and predicts poor prognosis in hepatocellular carcinoma. Oncogene 2016;35:1575-1584.

18 Lin L, Tu HB, Wu L, Liu M, Jiang GN: MicroRNA-21 Regulates Non-Small Cell Lung Cancer Cell Invasion and Chemo-Sensitivity through SMAD7. Cell Physiol Biochem 2016;38:2152-2162.

19 Li B, Shi XB, Nori D, Chao CK, Chen AM, Valicenti R, White Rde V: Down-regulation of microRNA 106b is involved in p21-mediated cell cycle arrest in response to radiation in prostate cancer cells. Prostate 2011;71:567-574.

20 Petrocca F, Visone R, Onelli MR, Shah MH, Nicoloso MS, de Martino I, Iliopoulos D, Pilozzi E, Liu CG, Negrini M, Cavazzini L, Volinia S, Alder H, Ruco LP, Baldassarre G, Croce CM, Vecchione A: E2F1-regulated microRNAs impair TGFbeta-dependent cell-cycle arrest and apoptosis in gastric cancer. Cancer Cell 2008;13:272-286.

21 Smith AL, Iwanaga R, Drasin DJ, Micalizzi DS, Vartuli RL, Tan AC, Ford HL: The miR-106b-25 cluster targets Smad7, activates TGF-beta signaling, and induces EMT and tumor initiating cell characteristics downstream of Six1 in human breast cancer. Oncogene 2012;31:5162-5171.

22 Cai K, Wang Y, Bao X: MiR-106b promotes cell proliferation via targeting RB in laryngeal carcinoma. J Exp Clin Cancer Res 2011;30:73.

23 Li Y, Tan W, Neo TW, Aung MO, Wasser S, Lim SG, Tan TM: Role of the miR-106b-25 microRNA cluster in hepatocellular carcinoma. Cancer Sci 2009;100:1234-1242.

24 Ma N, Zhang W, Qiao C, Luo H, Zhang X, Liu D, Zang S, Zhang L, Bai J: The Tumor Suppressive Role of MiRNA509-5p by Targeting FOXM1 in Non-Small Cell Lung Cancer. Cell Physiol Biochem 2016;38:1435-1446.

25 Matsuda S, Rouault J, Magaud J, Berthet C: In search of a function for the TIS21/PC3/BTG1/TOB family. FEBS Lett 2001;497:67-72.

26 Deng B, Zhao Y, Gou W, Chen S, Mao X, Takano Y, Zheng H: Decreased expression of BTG3 was linked to carcinogenesis, aggressiveness, and prognosis of ovarian carcinoma. Tumour Biol 2013;34:2617-2624.

27 Lim IK: TIS21 (/BTG2/PC3) as a link between ageing and cancer: cell cycle regulator and endogenous cell death molecule. J Cancer Res Clin Oncol 2006;132:417-426.

-28 Du Y, Liu P, Zang W, Wang Y, Chen X, Li M, Zhao G: BTG3 upregulation induces cell apoptosis and suppresses invasion in esophageal adenocarcinoma. Mol Cell Biochem 2015;404:31-38.

29 Chen X, Chen G, Cao X, Zhou Y, Yang T, Wei S: Downregulation of BTG3 in non-small cell lung cancer. Biochem Biophys Res Commun 2013;437:173-178.

-30 Ren XL, Zhu XH, Li XM, Li YL, Wang JM, Wu PX, Lv ZB, Ma WH, Liao WT, Wang W, Ding YQ, Liang L: Downregulation of BTG3 promotes cell proliferation, migration and invasion and predicts survival in gastric cancer. J Cancer Res Clin Oncol 2015;141:397-405.

-31 Lin TY, Cheng YC, Yang HC, Lin WC, Wang CC, Lai PL, Shieh SY: Loss of the candidate tumor suppressor BTG3 triggers acute cellular senescence via the ERK-JMJD3-p16(INK4a) signaling axis. Oncogene 2012;31:3287-3297.

-32 Varkonyi-Gasic E, Wu R, Wood M, Walton EF, Hellens RP: Protocol: a highly sensitive RT-PCR method for detection and quantification of microRNAs. Plant Methods 2007;3:12.

-33 Chen W, Zheng R, Zeng H, Zhang S, He J: Annual report on status of cancer in China, 2011. Chin J Cancer Res 2015;27:2-12.

-34 Calin GA, Croce CM: MicroRNA signatures in human cancers. Nat Rev Cancer 2006;6:857-866.

-35 Esquela-Kerscher A, Slack FJ: Oncomirs - microRNAs with a role in cancer. Nat Rev Cancer 2006;6:259-269.

-36 Huang C, Ma R, Yue J, Li N, Li Z, Qi D: MiR-497 Suppresses YAP1 and Inhibits Tumor Growth in Non-Small Cell Lung Cancer. Cell Physiol Biochem 2015;37:342-352. 


\section{Cellular Physiology Cell Physiol Biochem 2017;44:1545-1558

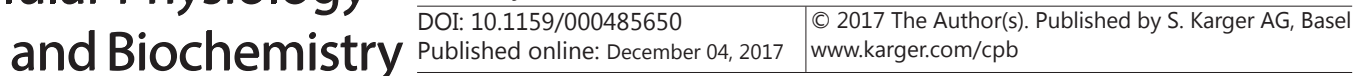

Wei et al.: MiR-106b-5p in NSCLC

37 Yan A, Yang C, Chen Z, Li C, Cai L: MiR-761 Promotes Progression and Metastasis of Non-Small Cell Lung Cancer by Targeting ING4 and TIMP2. Cell Physiol Biochem 2015;37:55-66.

38 Ma T, Zhao Y, Wei K, Yao G, Pan C, Liu B, Xia Y, He Z, Qi X, Li Z, Wang J, Shao Y: MicroRNA-124 Functions as a Tumor Suppressor by Regulating CDH2 and Epithelial-Mesenchymal Transition in Non-Small Cell Lung Cancer. Cell Physiol Biochem 2016;38:1563-1574.

-39 Yau WL, Lam CS, Ng L, Chow AK, Chan ST, Chan JY, Wo JY, Ng KT, Man K, Poon RT, Pang RW: Over-expression of miR-106b promotes cell migration and metastasis in hepatocellular carcinoma by activating epithelialmesenchymal transition process. PLoS One 2013;8:e57882.

-40 Liu F, Gong J, Huang W, Wang Z, Wang M, Yang J, Wu C, Wu Z, Han B: MicroRNA-106b-5p boosts glioma tumorigensis by targeting multiple tumor suppressor genes. Oncogene 2014;33:4813-4822.

-41 Li N, Liu Y, Miao Y, Zhao L, Zhou H, Jia L: MicroRNA-106b targets FUT6 to promote cell migration, invasion, and proliferation in human breast cancer. IUBMB Life 2016;68:764-775.

42 Zhang GJ, Li JS, Zhou H, Xiao HX, Li Y, Zhou T: MicroRNA-106b promotes colorectal cancer cell migration and invasion by directly targeting DLC1. J Exp Clin Cancer Res 2015;34:73.

-43 Xiang W, He J, Huang C, Chen L, Tao D, Wu X, Wang M, Luo G, Xiao X, Zeng F, Jiang G: mi R-106b-5p targets tumor suppressor gene SETD2 to inactive its function in clear cell renal cell carcinoma. Oncotarget 2015;6:4066-4079.

44 Guehenneux F, Duret L, Callanan MB, Bouhas R, Hayette S, Berthet C, Samarut C, Rimokh R, Birot AM, Wang Q, Magaud JP, Rouault JP: Cloning of the mouse BTG3 gene and definition of a new gene family (the BTG family) involved in the negative control of the cell cycle. Leukemia 1997;11:370-375.

45 Yanagida S, Taniue K, Sugimasa H, Nasu E, Takeda Y, Kobayashi M, Yamamoto T, Okamoto A, Akiyama T: ASBEL, an ANA/BTG3 antisense transcript required for tumorigenicity of ovarian carcinoma. Sci Rep 2013;3:1305.

-46 Majid S, Dar AA, Ahmad AE, Hirata H, Kawakami K, Shahryari V, Saini S, Tanaka Y, Dahiya AV, Khatri G, Dahiya R: BTG3 tumor suppressor gene promoter demethylation, histone modification and cell cycle arrest by genistein in renal cancer. Carcinogenesis 2009;30:662-670.

-47 Majid S, Dar AA, Shahryari V, Hirata H, Ahmad A, Saini S, Tanaka Y, Dahiya AV, Dahiya R: Genistein reverses hypermethylation and induces active histone modifications in tumor suppressor gene B-Cell translocation gene 3 in prostate cancer. Cancer 2010;116:66-76.

-48 Miyai K, Yoneda M, Hasegawa U, Toita S, Izu Y, Hemmi H, Hayata T, Ezura Y, Mizutani S, Miyazono K, Akiyoshi K, Yamamoto T, Noda M: ANA deficiency enhances bone morphogenetic protein-induced ectopic bone formation via transcriptional events. J Biol Chem 2009;284:10593-10600.

49 Yoshida Y, Hosoda E, Nakamura T, Yamamoto T: Association of ANA, a member of the antiproliferative Tob family proteins, with a Caf1 component of the CCR4 transcriptional regulatory complex. Jpn J Cancer Res 2001;92:592-596.

50 Ou YH, Chung PH, Hsu FF, Sun TP, Chang WY, Shieh SY: The candidate tumor suppressor BTG3 is a transcriptional target of p53 that inhibits E2F1. EMBO J 2007;26:3968-3980.

51 Lv Z, Zou H, Peng K, Wang J, Ding Y, Li Y, Ren X, Wang F, Chang R, Liang L, Ding Y: The suppressive role and aberrent promoter methylation of BTG3 in the progression of hepatocellular carcinoma. PLoS One 2013;8:e77473. 